\title{
NOTA DI RETTIFICA (SULL'EPIGRAMMA GRECO DELLA COLONNA ALESSANDRINA DI DINOCRATE)
}

\author{
XAVIER ESPLUGA \\ Universitat de Barcelona \\ xespluga@ub.edu \\ ORCID: 0000-0002-6690-197
}

In un recente contributo dedicato all'analisi complessiva del ms. Vat. Lat. 3616, autografo di Felice Feliciano, ${ }^{1}$ è stata erratamente ritenuta inedita un'iscrizione spuria, contenuta in questa silloge epigrafica (a c. 68r), posta alla base della colonna innalzata ad Alessandria da Dinocrate, l'architetto rodio che disegnò la nuova capitale dell'Egitto per ordine di Alessandro Magno. ${ }^{2}$ Benché essa non compaia, accolta naturalmente tra le false, nei corpora epigrafici più recenti, l'iscrizione ha alle sue spalle una lunghissima tradizione, sia per il testo greco, ritenuto una creazione di Ciriaco d'Ancona, ${ }^{3}$ sia per le diverse traduzioni latine che, a volte, l'accompagnano. ${ }^{4}$ In più, questa tradizione spuria si mescola con quella di un'altra iscrizione greca, questa volta genuina (CIG 4681), ${ }^{5}$ posta alla base della colonna detta 'di Pompeo' presente ancor'oggi nella città egiziana. Quest'ultima, nota ai viaggiatori di età moderna, ricorda un'onorificenza per Diocleziano da parte di un prefetto d'Egitto di identificazione controversa (probabilmente un Aelius Publius, documentato nel 298/299).

Il ricordo della colonna compare già nei resoconti delle gite di Ciriaco d'Ancona, il quale si recò in Egitto in tre occasioni, ${ }^{6}{ }^{1}$ 'ultima delle quali si pone nel settembre

\footnotetext{
${ }^{1}$ Espluga 2019: part. p. 212, nr. c). Il manoscritto è integralmente riprodotto nel sito digitale della Biblioteca Apostolica Vaticana.

${ }^{2} \mathrm{Il}$ personaggio è noto da alcune fonti classiche, già conosciute nel XV secolo, come ad esempio, VITR. 2 praef:: iussim eum (sc. Dinocratem) suo nomine ciuitatem Alexandriam constituere; AMM. MARC. 22.16: Alexandria enim vertex omnium est civitatum, quam multa nobilitant, et magnificentia conditoris altissimi et architecti soltertia Dinocratis ...; PLIN. nat. 7.38: Dinocrates metatus Alexandro condente in Aegypto Alexandriam; SOLIN. 320: Alexandriam et operis ipsius magnitudo et auctor Macedo nobilitant: quam metatus Dinocrates architecton alterum in conditore in memoria locum detinet.

${ }^{3}$ Micheli (1981: 81).

${ }^{4}$ Limitandosi solo ai principali corpora epigrafici, si veda Apianus, Amantius 1534: 511, Gruter 1602: 1020, nr. 9, Gruter 1607: 1020, nr. 9, Gruter 1707: 186, nr. 2, Boldoni 1660: 568, Muratori 1739: CMXLIX, nr. 6, Maffei 1765: cc. 79-80, Osann 1832: 229-351; introduzione a CIG IV 4681, CIL III, p. 3, nr. 2; Micheli 1981: 81, Giuliano 1983: 30-31, tav. V, fig. 1.

${ }^{5}$ Oltre a CIG IV 4681, si veda anche Dittenberger 1905: 458-460, nr. 718; $S B \mathrm{~V}, \mathrm{nr} .8278$ et add. p. 546; IGRRP, p. 369, nr. 1068; Vandersleyen 1958; SEG XVII, 789; Fraser 1959: 93, nr. 23; SEG XVIII, 643; Vandersleyen 1972: 66-70; Fraser 1972: 85, nr. 10; Wagner, Gascou 1978; Bernand 1983: 21, nr. 1068; Kayser 1994: 52-57, nr. 15.

6 Per i viaggi egiziani di Ciriaco, si veda Lumbroso 1879: 429, Van Essen 1956, Van Essen 1958. Si veda anche Dannenfeld 1959, Weiss 1966: 323-337, Castelli 1979: 3, nota 132, Williams Lehmann 1977.
} 
di un anno che è fissato al 1436 (cronologia tradizionale) ovvero al 1437 (cronologia proposta da Essen). ${ }^{7}$ Le notizie di quest'ultima visita si trovano in diversi materiali derivati, direttamente o indirettamente, dall'anconitano. Così, la tappa alessandrina è presente nella biografia ciriacana del conterraneo Francesco Scalamonti, conservata nel noto manoscritto della Biblioteca Capitolare di Treviso, di mano di Felice Feliciano; ${ }^{8}$ la si legge anche nella lettera ad Eugenio IV, del 18 ottobre 1441, la cui versione più completa è conservata nel ms. Ott. Lat. 2967, cc. 1r-20v (part. c. 20rv), codice di mano di Filippino Bononi. ${ }^{9}$ In entrambi i documenti si fa riferimento alla colonna alessandrina, che già all'epoca di Ciriaco veniva chiamata 'Pompeiana'. ${ }^{10}$

\begin{tabular}{|l|l|}
\hline Vita Kyriaci Anconitani & Lettera ad Eugenio IV \\
\hline $\begin{array}{l}\text { Ubi primum ingentia Phariae praecelsae olim } \\
\text { turris vestigia, regias ex Numidico lapide }\end{array}$ & $\begin{array}{l}\text { Extra ciuitatis muros pipeream prope portam } \\
\text { vortas, maximum }\end{array}$ \\
\hline et magnam ex Dinocrate architecto Alexandri & maximam illam columnam, quam incertus \\
Macedonis columnam, quae (sic) hodie uulgus & vulgus hodie Pompeianam appellat, et nos \\
Pompeianam apellat, inspexit. ${ }^{11}$ & vero Alexandricam regis. \\
\hline & $\begin{array}{l}\text { Dinocratem nobilem architectum eximiam per } \\
\text { basim antiquo ex epigrammate novimus } \\
\text { erexisse. }{ }^{12}\end{array}$ \\
\hline
\end{tabular}

Tavola I: Ricordi della gita alessandrina di Ciriaco

Ciriaco allude, senza riportarne il testo, ad un epigramma 'parlante' che dichiara aver visto («per basim ... ex epigrammate ... nouimus»), in cui si attribuisce la costruzione della colonna a Dinocrate, l'architetto di Alessandro Magno. La lettera ad Eugenio IV, del 1441 riflette la fraseologia dell'epigramma greco, perché vi sono paralleli tra le formule di quest'ultimo, noto solo da manoscritti più tardi, e la redazione latina della lettera: $\pi \varepsilon \rho \iota \kappa \lambda \iota t o ́ \varsigma=$ nobilem; $\alpha \rho \chi \imath \varepsilon \dot{\varepsilon} \kappa \tau o \varsigma$ (sic) = architectum; ó $\rho \theta o \sigma \varepsilon v($ sic $)=$ erexisse.

Il testo dell'epigramma greco con una traduzione latina doveva essere presente nell'antigrafo da cui fu vergato il ms. 1191 della Biblioteca Palatina di

\footnotetext{
${ }^{7}$ Quest'ultimo anno è il preferito da Van Essen 1958: 293, nota 1.

8 Treviso, Biblioteca Capitolare, ms. I.138 (37). La vita è stata edita in numerose occasioni, a cominciare da Colucci (1792) e più recentemente in Mitchell, Bodnar 1996: 31, § 17. Estratti della gita egiziana, con particolare riguardo all'elefante e alla giraffa, si conservano in altre lettere mandate da Ciriaco ai suoi amici e corrispondenti.

${ }_{9}^{9}$ Città del Vaticano, Biblioteca Apostolica Vaticana, ms. Ott. Lat. 2967, cc. 1r-20v, part. c. 20 rv. Il testo fu edito da Mehus 1742: cc. 48-50.

10 Per questo monumento alessandrino, si veda Adriani 1966: 97-98; Thiel 2006.

11 Treviso, Biblioteca Capitolare, ms. I.138 (37), c. 26r (si adopera la cartolazione coeva). Si segnala che nel margine in corrispondenza del nome Feliciano è stata aggiunta la postilla: Dinocrates architectus.

12 Città del Vaticano, Biblioteca Apostolica Vaticana, ms. Ott. Lat. 2967, c. 20rv.
} 
Parma, uno dei testimoni primari della gita orientale di Ciriaco d'Ancona. ${ }^{13}$ Nelle cc. 59-67 di quest'ultimo codice si conserva un particolare raggruppamento di iscrizioni di centri orientali (Alessandria, la fortezza di Babilonia nei pressi di Cairo, Pergamo, Smirne, Cizico, Salonicco, Costantinopoli) che, per comodità, sarà denominata la 'sezione delle meraviglie orientali', il cui materiale è derivato in parte dalle ricerche antiquarie di Ciriaco d'Ancona e in parte dai suoi viaggi orientali. ${ }^{14}$

Queste carte hanno la particolarità che la mano intervenuta - responsabile della maggior parte del manoscritto - non ha vergato buona parte dei testi in caratteri greci, così come ha ugualmente omesso le eventuali illustrazioni che potevano accompagnare alcune epigrafi. Questa sezione contiene, dunque, le didascalie introduttive ai testi 'epigrafici', in rosso, e alcune delle traduzioni latine dei testi greci, in inchiostro scuro. La prima delle meraviglie orientali, in c. 59r, è appunto la colonna alessandrina di Dinocrate, di cui è rimasta la didascalia introduttiva (in latino) (1a), lo spazio in bianco destinato alla trascrizione del testo greco dell'epigramma (1b), e la traduzione latina (1c) (Tavola II).

\begin{tabular}{|c|c|c|}
\hline Nr. & Carta & Testo \\
\hline $1 a$ & $59 r, 1$ & $\begin{array}{l}\text { Apud Alexandriam egypti ad basim ex unico Numidico lapide sub columna } \\
\text { ingenti in Allexandru }(m) \text { regem et Dinocretem architecton suu }(m) \text { illust }(r) \text { em }\end{array}$ \\
\hline $1 b$ & $59 r, 2$ & 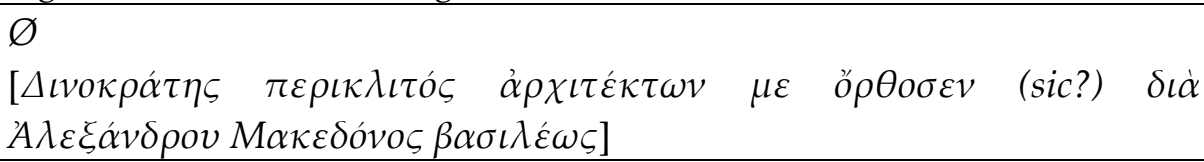 \\
\hline 1c & $59 r, 3$ & $\begin{array}{l}\left.\text { Dinocrates }{ }^{2} \text { (Dinocretes }{ }^{1}\right) \text { nobilissimus architectus me erexit cum Alexandro } \\
\text { Macedone rege }\end{array}$ \\
\hline
\end{tabular}

Tavola II: Didascalia e traduzione dell'epigramma della colonna alessandrina (Parma, Biblioteca Palatina, ms. 1191, c. 59r)

La didascalia è una sintesi delle informazioni riportate nelle due fonti precedenti, la Vita ciriacana di Scalamonti e la lettera ad Eugenio IV, in quanto si dà la localizzazione ( $A$ Apud Alexandriam egypti»), il supporto («ad basim»), il materiale («ex unico Numidico lapide»), la posizione («sub columna ingent»i) e parte del contenuto dell'epigramma, aggiungendo il ricordo del re macedone («cum Alexandro Macedone rege») e del suo architetto («Dinocrates nobilissimus architectus», con la giusta trascrizione del nome di quest'ultimo).

È, dunque, possibile ricostruire il testo greco non soltanto da questa traduzione latina del codice parmense, da attribuire a Ciriaco, ma anche dalla

13 Per questo manoscritto, si veda Kristeller 1977: 40. Sul ruolo di questo manoscritto, si veda CIL III, p. XXII; CIL V, pp. 35, 78, 163, 173, 624; CIL VI, p. XLI; Henzen 1866: part. 762 e passim; ICVR II/1, p. 359; Ziebarth 1905: part. pp. 188-191 (con le tavole annesse); Bodnar 1960: 101-110.

14 Per una biografia di sintesi dell'anconitano, si veda Colin 1981. Per l'edizione della biografia di Scalamonti e delle lettere, si veda Mitchell, Bodnar, Foss 2015; Mitchell, Bodnar 1996; Bodnar 1960; Bodnar, Mitchell 1976; Bodnar, Foss 2003. Per la raccolta di materiale greco, si veda Chatzidakis 2017. 
presenza del testo greco in manoscritti più tardi, di mano di Feliciano, come lo stesso Vat. Lat. 3616, o in copie di suoi autografi come un noto taccuino, certamente di derivazione felicianea, conservatosi nella Biblioteca della Sovrintendenza alle Gallerie di Firenze. In questo codice, dopo la didascalia "Apud Alexandria(m) egipti i(n) Colu(m)na numidica mire magnitudinis», l'epigramma è stato inciso nel fusto di una colonna (Figura 1$) \cdot{ }^{15}$ In tutti questi casi compare la lezione $\Delta \eta \mu о \kappa \rho \alpha ́ \tau \eta \varsigma$ per il nome dell'architetto rodio e la variante $\dot{\alpha} \rho \chi \iota \tau \dot{\varepsilon} \kappa \tau o \varsigma$, al posto della forma $\dot{\alpha} \rho \chi \iota \tau \dot{\varepsilon} \kappa \tau \omega \nu$, propria del greco classico.

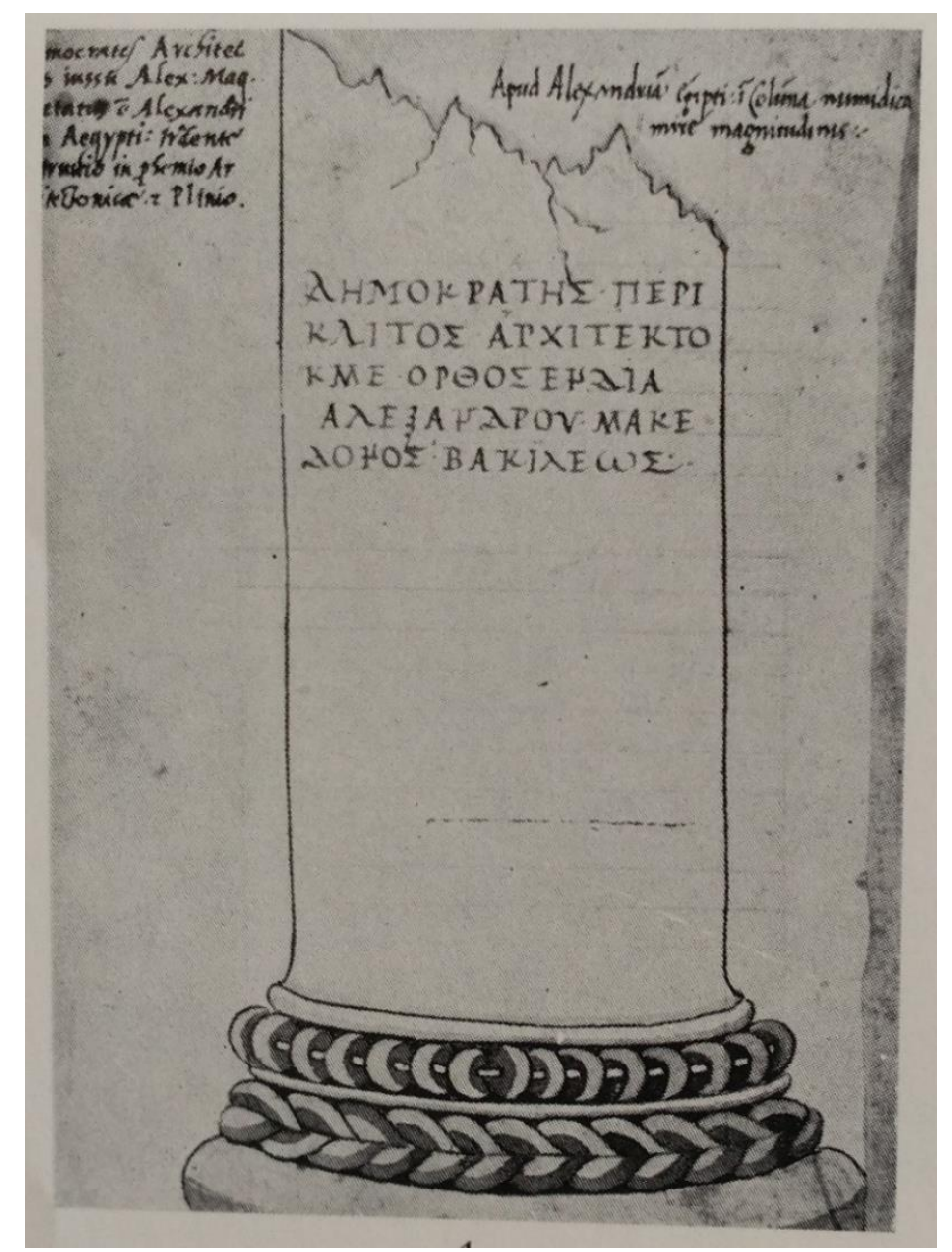

Figura 1: L'iscrizione della colonna alessandrina di Dinocrate [olim Firenze, Biblioteca della Sovrintendenza alle Gallerie, ms. V.2.7.a, c. 67r (da Giuliano 1983: tav. v, fig. 1)]

Oltre a questi testimoni manoscritti tardo quattrocenteschi, l'epigramma si legge, introdotto dalla didascalia: «Apud Alexandriam Aegypti», in alcuni degli esemplari della silloge epigrafica di Giovanni Giocondo, sia quelli della prima recensio, come documenta il ms. 270 della Biblioteca Capitolare di Verona

${ }^{15}$ Firenze, Biblioteca della sovrintendenza alle Gallerie di Firenze, ms.V.2.7.a, c. 67v, edito da Micheli 1981: 81 (con l'errore numidia). Per la bibliografia su questo manoscritto, si veda CIL IX-X ind. auct. s.v. Codex musei Florentini 7a; ICVR II, p. 343 e pp. 403-404; Micheli 1981; Giuliano 1983, con un'immagine della colonna nella tav. V, fig. 1. Si segnala che nel testo greco, il sigma finale di APXITEKTO $\Sigma$ è stata confusa con una kappa (APXITEKTOIK). 
(rappresentante appunto di questa recensio), ${ }^{16}$ sia quelli della seconda recensio, come il ms. Magl. XXVIII, 5 della Biblioteca Nazionale Centrale di Firenze. ${ }^{17}$

Da queste fonti manoscritte l'epigramma arrivò fino alle opere a stampa. Sempre da una silloge epigrafica tardo quattrocentesca fu presa la notizia sull'iscrizione che compare nei Commentaria del volterrano Raffaele Maffei $\left(†\right.$ 1522), ${ }^{18}$ pubblicati nel 1506. Maffei ricorda la realizzazione dell'architetto (segnando l'errore presente nel suo nome) nella città di Alessandria e riporta anche il testo dell'iscrizione. Nonostante ciò, Maffei cambia il verbo principale, reso ora $\check{\varepsilon} \theta \varepsilon \sigma \varepsilon v$, forma che traduce il latino metatus est, usato da Plinio e da Solino e che fa riferimento all'impegno dell'architetto rodio. Così, la voce parlante dell'epigramma non è più la colonna, bensì la stessa città di Alessandria:

Democrates Architectus Alexandriq(ue) Alexandria(m) metatus est in qua urbe huiuscemodi de eo

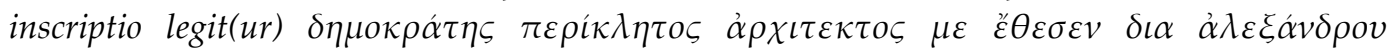
$\mu \alpha \kappa \varepsilon \dot{\varepsilon} \omega v o \varsigma$. Ex quo apparet corrupte a quibusda $(m)$ scribi Dinocrates Democrates palestrita facto in statio circulo co(n)sistens a nullo dimoueri poterat. ${ }^{19}$

Da uno di questi manoscritti tardo quattrocenteschi, circolanti in area tedesca, l'epigramma passò alle monumentali Inscriptiones Sacrosanctae Vetustatis di P. Apiano (Peter Bienewitz) e B. Amanzio (Bartholomäus Pelten), stampate a Ingolstadt nel 1534 (Figura 2). ${ }^{20}$

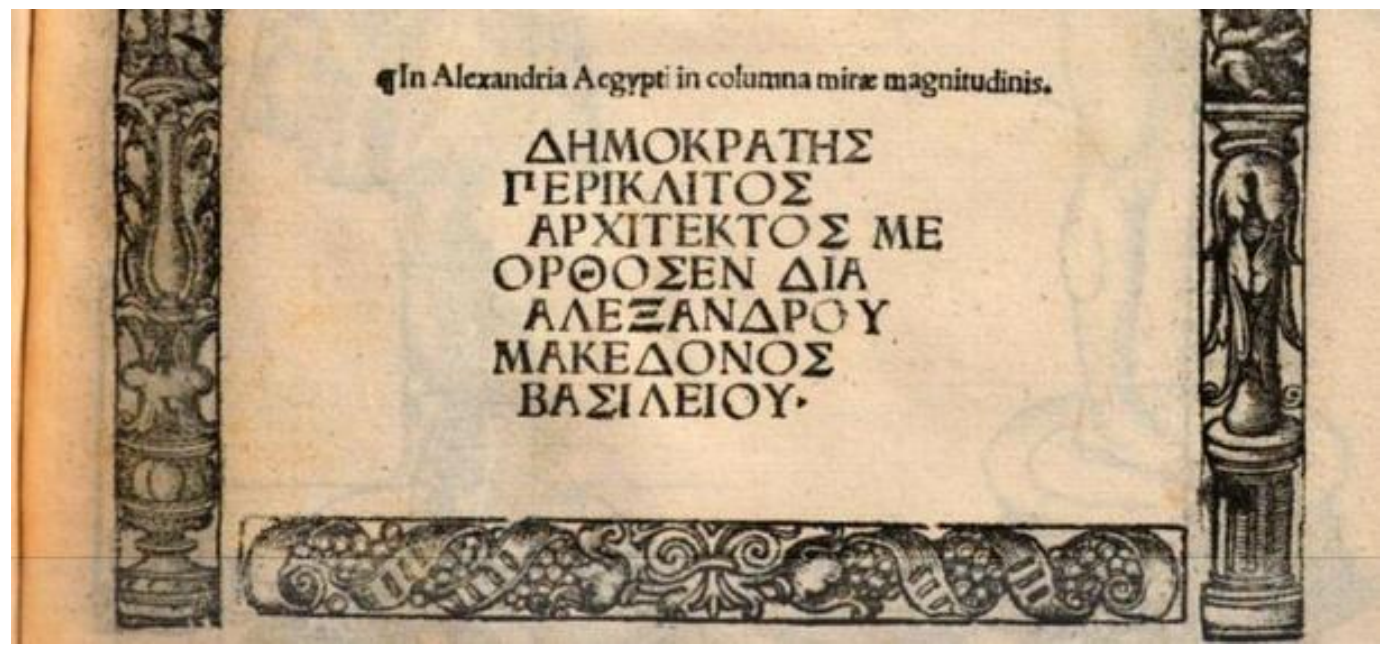

Figura 2: L'iscrizione della colonna alessandrina di Dinocrate [da Apiano, Amanzio 1534: 511]

\footnotetext{
16 Verona, Biblioteca Capitolare, ms. 270, c. 168v (adopero la cartolazione coeva del codice). Per questo manoscritto, vergato da Bartolomeo Sanvito, si veda De la Mare, Nuvoloni 2009: 314-315, nr. 96.

17 Firenze, Biblioteca Nazionale Centrale, ms. Magl. XXVIII, 5, c. 206v (adopero la cartolazione coeva del codice). Per questo codice, parimenti vergato da Sanvito, si veda De la Mare, Nuvoloni 2009: 332, nr. 104.

18 Per questo personaggio, si veda Benedetti 2006.

${ }^{19}$ Maffei 1506: 207 (libro XV).

${ }^{20}$ Apiano, Amanzio 1534: 511.
} 
Nelle Inscriptiones di Apiano, il testo greco dell'iscrizione (che compare con la variante $\beta \alpha \sigma \iota \lambda \varepsilon \iota \sigma \tilde{v}$ invece di $\beta \alpha \sigma \iota \lambda \varepsilon \omega \varsigma)$ è preceduto dalla didascalia «In Alexandria Aegypti in columna mirae magnitudinis», che riecheggia quella del taccuino fiorentino.

Le Inscriptiones di Apiano sono la fonte di buona parte della tradizione successiva. ${ }^{21}$ In effetti, dalla magna opera di Apiano e Amanzio il testo greco dell'iscrizione fu successivamente ripreso dai diversi corpora sei- e settecenteschi a cominciare dal corpus del Grutero. Così, l'epigramma è accolto nell'edizione gruteriana del 1602, preceduta dalla didascalia «In Alexandria Aegypti in columna mirae magnitudinis» che rivela la dipendenza da Apiano, esplicitamente dichiarata («Ex Apiano») (Figura 3a), ${ }^{22}$ nell'edizione gruteriana del 1607, curata da J.J. Graevius, fu aggiunta una lunga nota in cui si alludeva al corretto nome dell'architetto (con il riferimento alle principali fonti classiche in cui veniva citato); ${ }^{23}$ per ultimo, anche nell'edizione gruteriana del 1707, curata da Pieter Burman, l'edizione del testo è modificata in diversi punti: l'aggettivo applicato

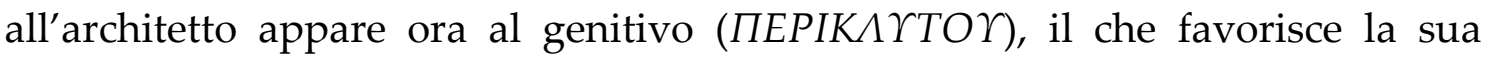
interpretazione come patronimico; la forma verbale dell'aoristo è scritta con omega iniziale ( $\omega \rho \theta \omega \sigma \varepsilon v)$, come converrebbe; d'altra parte, però, si è omessa la denominazione regale presente alla fine del testo (Figura $3 b) .{ }^{24}$
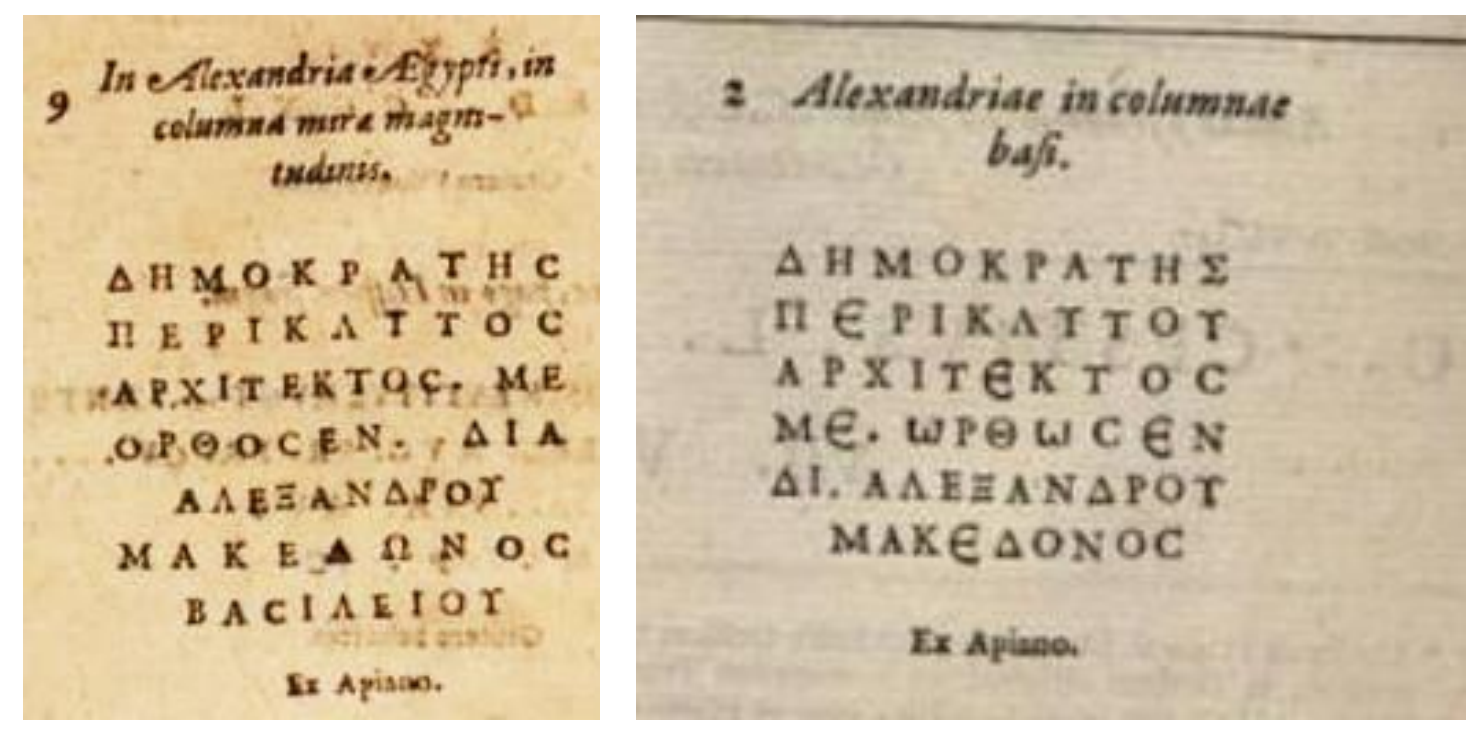

Figura 3: L'iscrizione della colonna alessandrina di Dinocrate [da Gruter 1602: 1020, nr. 9 -a destra - e Gruter 1707: 186, nr. 2 - a sinistra]

L'epigramma è ricordato con una localizzazione quasi identica a quella gruteriana («Item in Aegypto eadem lingua inscribitur Columna mirae altitudinis»)

${ }^{21}$ Dalle Inscriptiones fu successivamente preso da diversi autori: Kiechel 1972: 223; Quaresmio 1639: 1010.

${ }^{22}$ Gruter 1602: 1020, nr. 9.

${ }^{23}$ Gruter 1607: 1020, nr. 9.

${ }^{24}$ Gruter 1707: 186, nr. 2. 
nell'opera di Ottavio Boldoni (1660), che offre il testo greco, con le varianti di Apiano, ma preso, probabilmente, dalle prime edizioni del corpus di Grutero, citato a continuazione; si legge anche una nuova traduzione latina («Democrates Nobilis Architectus me erexit pro Alexandro Macedone Rege»), che forse è opera dello stesso Boldoni..$^{25}$

Il Muratori edita il testo greco nel secondo volume del suo Novus Thesaurus (1739). ${ }^{26}$ Nell'edizione muratoriana furono adoperate due fonti: da una parte, il manoscritto magliabecchiano di Giocondo, sopra citato, allora proprietà di Antonfrancesco Marmi, per intermediazione di Anton Francesco Gori che comunicò il testo all'erudito modenese; dall'altra, fu anche adoperato il corpus del Grutero nell'edizione del 1707. Oltre al testo greco, Muratori aggiunse una nuova traduzione latina («Democrates Celebris Architectus Me Erexit Jubente Alexandro Macedone Rege»).

Il primo autore a ritenere spuria quest'iscrizione è il conte veronese Scipione Maffei nella sua Artis critica lapidariae. ${ }^{27}$ Costui prende il testo dall'edizione gruteriana del 1707, perché intrepreta il genitivo ПEPIK $\Lambda$ ҮTO come un patronimico, tradotto come «Perycliti f.» all'interno di una nuova versione latina («Democrates Periclyti F. me erexit propter Alexandrum Macedonem»).

In ogni caso, l'analisi più approfondita sull'epigramma greco è il lungo contributo di F. Osann, intitolato De columna Alexandrina, apparso nel primo numero delle neonate Memorie dell'Istituto di Corrispondenza Archeologica, del 1832. ${ }^{28}$ Osann fu il primo a mettere in rapporto la colonna di Pompeo, vista e descritta dai viaggiatori moderni, con il testimone di Ciriaco. ${ }^{29}$ Il filologo tedesco aveva conosciuto il brano ciriacano a partire dell'excerptum che Jean-BaptisteGaspard d'Ansse de Villoison (Villoisonus) aveva pubblicato all'inizio dell'Ottocento. Di fatto il grecista francese si era occupato dal testo greco della colonna di Pompeo, sia nel 1803, in una prima riposta alla lettura dell'iscrizione fatta da Jaubert (quando non conosce ancora il testo di Ciriaco), ${ }^{30}$ sia nel 1809, in connessione con il racconto di Ciriaco, che conobbe tramite l'Itinerarium del Mehus. $^{31}$

Tutte queste notizie sono in parte confluite nel nr. 4681 del terzo volume del Corpus Inscriptionum Graecarum di August Boeckh, curato da J. Franz e apparso

${ }^{25}$ Boldoni 1660: 586: «Item in Aegypto eadem lingua (sc. Graeca) inscribitur Columna mirae altitudinis:

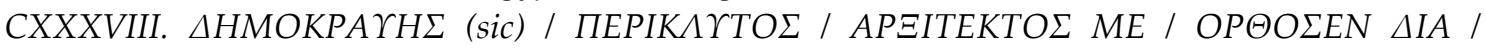

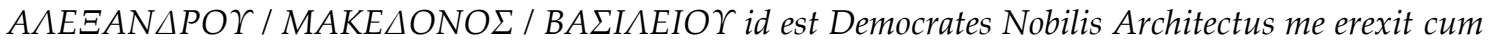
Alexandro Macedone Rege».

${ }_{26}$ Muratori 1739: p. CMXLIX, nr. 6. Il Muratori è la fonte dei Commentaria 1786: p. 266.

${ }^{27}$ Maffei 1765: cc. 79-80. Si ricordi che quest'opera di Maffei era anche stata inclusa nel Supplementum muratoriano di Donati 1765.

28 Osann 1832.

29 Osann 1832: 237.

${ }^{30}$ D'Ansse de Villoison 1803. Sul personaggio, si veda Calvié 2017.

${ }^{31}$ D'Ansse de Villoison 1809: part. p. 316-317, nota e. 
nel 1848. In quest'opera, prima di editare l'iscrizione della base della colonna di Pompeo, Franz ricorda alcune tappe della tradizione dell'epigramma, riportando l'edizione del textus receptus (da Apiano) e suggerendo una proposta di restituzione fondata su diverse correzioni che comprendono la restituzione del corretto nome dell'architetto rodio ( $\Delta \varepsilon \iota v o \kappa \rho \alpha ́ \tau \eta \zeta)$, del suo ufficio (nella forma classica $\dot{\alpha} \rho \chi \iota \tau \dot{\varepsilon} \kappa \tau \omega v$ ), nonché del titolo regale di Alessandro (ora reso come $M \alpha \kappa \varepsilon \delta o ́ v \omega v \beta \alpha \sigma \iota \lambda \varepsilon \omega \varsigma)$. Si è però mantenuta la forma del genitivo di $\Pi \varepsilon \rho \iota \kappa \lambda \varepsilon \iota \tau o \tilde{v}$. Osann suggerì inoltre che questo epigramma spurio era sorto da una cattiva lettura dell'iscrizione greca tuttora esistente, eseguita da Ciriaco, successivamente sfruttata da un fraudolento ignoto che diede vita al falso:

Nam homo nescio quis ... arrepta illa notitia Cyriaci quum in eius schedis non repperisset antiquum illus epigramma, Marte suo procudit titulum, translatis verbis Cyriaci et compositis in ordine quendam: Dinocrates nobilis architectus $=\triangle E I N O K P A T H \Sigma($ uel $\triangle H M O K P A T H \Sigma)$; erexit $=M E$ $\Theta P \Theta \Omega \Sigma E N$; Alexandri regis Macedonoum $=\triangle I A$ A $E E A N \triangle P O \Upsilon M A K E \triangle O N \Omega N$.

Il materiale qui raccolto illustra le vicissitudini di una delle tante ideazioni di Ciriaco che cercavano di far rivivere, con una certa naïveté, le meraviglie e i personaggi illustri dell' Antichità Classica grazie alla memoria epigrafica. In ogni caso serva questo contributo come palinodia personale per aver, incautamente, preso per inedito questo 'falso' ciriacano.

\section{BIBLIOGRAFIA}

Adriani, A. (1966), Repertorio d'Arte dell'Egitto Greco-Romano. Serie C, vol. I, Palermo, Fondazione 'Ignazio Mormino' del Banco di Sicilia.

APIANO, P., AMANTIUS, B. (1534), Inscriptiones sacrosanctae vetustatis ..., Ingolstadt, in aedibus P. Apiani, 1534.

BenedetTI, S. (2006), «Maffei, Raffaele», in Dizionario Biografico degli Italiani, vol. 67, Roma, Istituto dell'Enciclopedia Italiana.

BERNARD, É. (1983), Inscriptions grecques d'Égypte et de Nubie: répertoire bibliographique des IGRR, Besançon, Université de Franche-Comté.

BODNAR, E. W., FOSS, C. (2003), Cyriac of Ancona, Later travels. Edited and translated by Edward W. Bodnar with Clive Fosse, Cambridge (Mass.), Harvard University Press (The I Tatti Renaissance library, 10).

Bodnar, E. W., Mitchell, Ch. (1976), Cyriacus of Ancona's journeys in the Propontis and the Northern Aegean 1444-1445, ed. by E.W. Bodnar, Ch. Mitchell, Philadelphia, American Philosophical Society (Memoirs of the American Philosophical Society, 112).

BOLDONI, O. (1660), Epigraphica sive Elogia inscriptionesque quoduis genus pangendi ratio ..., Augustae Perusiae, ex Typografia Cameraili et Episcopali, apud Bartolos et Angelum Laurentium.

CAlviÉ, L. (2017), Jean-Baptiste-Gaspard D'Ansse de Villoison, 'Le Voyage à Venise'. La recherche de manuscrits grecs inédits à la fin du XVIIIe siècle suivi de 'Sur des recherches à faire dans le voyage de Constantinople et du Levant' de B. Monftaucon. Textes présentés, établis et annotés par Laurent Calvié, Toulouse, Anacharsis. 
CASTElli, P. (1979), I geroglifici e il mito dell'Egitto nel Rinascimento, Firenze, Edam (Contributi per la Storia delle Arti e della Cultura).

CHATZIDAKIS, M. (2017), Ciriaco d'Ancona und die Wiederentdeckung Griechenlands im 15. Jahrhundert, Petersberg, Michael Imhof-Mainz, Franz Philipp Rutzen (Studien zur Rezeption der Antike / Census of Antique Works of Art and Architecture Known in the Renaissance (Berlin-Brandenburgische Akademie der Wissenschaften / HumboldtUniversität zu Berlin), Winckelmann-Gesellschaft Stendal, Winckelmann-Institut der Humboldt-Universität zu Berlin, 2197-6406, 9).

Colin, J. (1981), Cyriaque d'Ancone. Le voyaguer, le marchand, l'humaniste, Paris, Maloine.

ColuccI, G. (1792), Vita di Ciriaco Anconitano scritta da Francesco Scalamonti tratta da un codice trivigiano e data in luce dall'Ab. Giuseppe Colucci premesso un commento, ed aggiuntevi le opportune annotazioni, in Delle Antichità Picene, vol. XV, Fermo, dai torchi dell'autore per G.A. Paccaroni.

D'ANSSE DE VILlOSION, J.-B.-G. (1803), «Palaeographie. fragment d'une Lettre de J.B.G D'Ansse de Villoison, membre de l'Institut national de France, au professeur Millin, sur l'inscription grecque de la prétendue colonne de Pompée», Magasin encyclopèdique ou Journal des Sciences, des Lettres et des Arts VIII an., 5, 55-60.

D'ANSSE DE VILLOSION, J.-B.-G. (1809), «Mémoire sur quelques inscriptions inconnues, ou publiées inexactement. Extrait de la relation du voyage littéaire fait dans le Levant», Mémoires de l'Académie Française, 47, 283-344.

DANNENFELD, K. H. (1959), «Egypt and Egyptian Antiquities in the Renaissance», Studies in the Renaissance 9, 7-27.

De la MARE, A. C., NuVOLONI, L. (2009), Bartolomeo Sanvito: the life \& work of a Renaissance scribe. Edited by Anthony Hobson \& Christopher de Hamel. With contributions by Scott Dickerson, Ellen Cooper Erdreich \& Anthony Hobson, Paris, Association internationale de bibliophilie (The handwriting of Italian humanists, II).

DitTENBERGER, W. (1905), Orientis Graeci Inscriptiones Selectae. Supplementum Sylloges Inscriptionum Graecarum, vol. II, Lipsiae, apud S. Hirzel.

DONATI, S. (1765), Novum Thesaurum Veterum Inscriptionum Cl. V. Ludovici Antonii Muratorii Supplementum collectore Sebastiano Donato, vol. I, Lucae, ex typografia L. Venturini.

EsPlUGA, X. (2019), «L'ultimo manoscritto epigrafico autografo di Felice Feliciano (Vat. lat. 3616)», Epigraphica, 81, 183-237.

FRASER, P. M. (1958), «Bibliography: Graeco-Roman Egypt Greek Inscriptions (1958)», Journal of Egyptian Archaeology, 45, 88-97.

Fraser, P. M. (1972), Ptolemaic Alexandria, vol. II, Oxford, Clarendon Press.

GIUliano, A. (1983), «La Fortuna di Alessandria in età moderna», in Alessandria e il mondo ellenistico-romano. Studi in onore di Achille Adriani, a cura di Nicola Bonacasa, Antonino Di Vita, (Studi e Materiali. Istituto di Archeologia. Università di Palermo, 4), Palermo, Università di Palermo.

GRUTER, J. (1602), Inscriptiones antiquae totius orbis Romani in absolutissimum corpus redactae ..., Heidelberg, ex officina Commeliniana.

GRUTER, J. (1607), Inscriptiones antiquae totius orbis Romani ..., vol. II, Amstelaedami, F. Halma.

GRUTER, J. (1707), Inscriptiones antiquae totius orbis Romani ..., vol. I, Amstelaedami, F. Halma. 
HENZEN, W. (1866), Über die von Cyriacus von Ancona gesammelten Inschriften der Stadt Rom, Monatsberichte der preußischen Akademie der Wissenschaften zu Berlin, 758-781

IGRRP (1911), Inscriptiones Graecae ad Res Romanas pertinentes, vol. I, Paris.

KAYSER, F. (1994), Recueil des Inscriptions grecques et latines (non funéraires) d'Alexandrie impériale, Le Caire, Institut français d'archéologie orientale du Caire.

KIECHEL, S. (1972), Voyages en Egypte pendant les années 1587-1588, éd. et trad. Serge Sauneron, Le Caire, Institut français d'archéologie orientale du Caire.

KRISTELleR, P. O. (1977), Iter Italicum. A Finding list of Uncatalogued or Incompletely Catalogued Humanistic MSS, Volume 2 Italy. Orvieto-Volterra, Vatican City, LeidenLondon, Brill, 1977.

Lumbroso, G. (1879), Descrittori italiani dell'Egitto e di Alessandria, Roma, R. Accademia dei Lincei (Memorie. Classe di Scienze Morali della R. Accademia dei Lincei, serie III, vol. 3).

MAFFEI, R. (1506), Commentariorum urbanorum octo et triginta libri ..., Roma per Joannem Besicken Alemanum.

MAFFEI, S. (1765), Clarissimi Viri Scipionis Maffei Marchionis Artis criticae lapidariae quae extant ex eiusdem autographo ..., Lucae, ex Typographiae Leonardi Venturini.

MeHUS, L. (1742), Kyriaci Anconitani Itinterarium, Florentiae, ex novo typographio Joannis Pauli Giovannelli.

MiCHELI, M. L. (1981), «Un taccuino di un ignoto umanista del XV secolo», Xenia, 6, 6382.

Mitchell, Ch., BODNAR, E. W. (1966), Vita viri clarissimi et famosissimi Kyriaci Anconitani by Francesco Scalamonti. Edited and translated by Charles Mitchell and Edward W. Bodnar, Philadelphia, American Philosophical Society.

Mitchell, Ch., BodNAR, E. W., Foss, C. (2015), Cyriac of Ancona. Life and early travels. Edited and translated by Charles Mitchell, Edward W. Bodnar and Clive Foss, Cambridge (Mass.)-London (England), Harvard University Press (I Tatti Renaissance library, 65).

MURATORI, L. A. (1739), Novus thesaurus veterum inscriptionum, vol. II, Mediolani, ex aedibus Palatini.

OsANN, F. G. (1832), «De columna Alexandrina Pompei nomine uulgo nominata», Memorie dell'Istituto di Corrispondenza Archeologica», 1, 229-351

QUARESMIO, F. (1639), Historica theologica et moralis Terrae Sanctae elucidatio ..., vol. II, Antverpiae, ex officina Plantiniana Balthasaris Moreti.

SB V [(1938)] = Sammelbuch Griechischer Urkunden aus Ägypten V, Heidelberg, Im Selbstverlag des Verfassers.

THIEL, W. (2007), «Die ,Pompeius-Säule' in Alexandria und die Vier-Säulen-Monumente Ägyptens», in Die Tetrarchie. Ein neues Regierungssystem und seine mediale Präsentation; [basiert auf dem Kolloquium des Lehr- und Forschungszentrums für die antiken Kulturen des Mittelmeerraumes der Universität zu Köln, 13.-14. Februar 2004] I herausgegeben von Dietrich Boschung und Werner Eck, Wiesbaden, Reichert (Schriften des Lehr- und Forschungszentrums für die antiken Kulturen des Mittelmeerraumes Centre for Mediterranean Cultures, ZAKMIRA 3).

VAN EsSEN, C. C. (1956), «I commentaria di Ciriaco d'Ancona», in Il mondo antico nel Rinascimento. Atti del V Convegno Internazionale di Studi sul Rinascimento, FirenzePalazzo Strozzi, 2-6 Settembre 1956, Firenze, G.C. Sansoni. 
VAN EsSEN, C. C. (1958), «Cyriaque d'Ancone en Egypte: fragments des Commentaria rerum antiquarum», Mededelingen der Koninklyke Nederlandse Akademie van Wetenschappen 12, 293-306.

VANDERSLEYEN, C. (1958), «Le préfet d'Égypte de la colonne de Pompée à Alexandrie», Chronique d'Égypte 33, 113-134.

VANDERSLEYEN, C. (1972), Chronologie des préfets d'Egypte de 284 à 395, Bruxelles, Latomus (Col. Latomus, 55).

WAGNER, G., GASCOU, J. (1978) «Nouvelles inscriptions grecques d'Égypte relevées par le père Sicard», Bulletin de l'Institut français d'archéologie orientale, 78, 259-260.

WEISS, R. (1966), «Ciriaco d'Ancona in Oriente», in Venezia e l'Oriente tra tardo Medioevo e Rinascimento, a cura di A. Pertusi, Firenze, G.C. Sanoni, 323-337.

Williams LeHMANN, P. (1977), Cyriacus of Ancona's Egyptian Visit and its Reflections in Gentile Bellini and Hieronymus Bosch, Locus Valley-New York, J.J. Augustin editorial.

ZIEBARTH, E. (1905), «De antiquissimis inscriptionum syllogis», Ephemeris Epigraphica, 9/2, 188-332. 